\title{
BMJ Open Bubble continuous positive airway pressure in the treatment of severe paediatric pneumonia in Malawi: a cost- effectiveness analysis
}

\author{
Teresa Bleakly Kortz, ${ }^{1}$ Benjamin Herzel, ${ }^{2}$ Elliot Marseille, ${ }^{3}$ James G Kahn ${ }^{4}$
}

To cite: Kortz TB, Herzel B, Marseille $\mathrm{E}$, et al. Bubble continuous positive airway pressure in the treatment of severe paediatric pneumonia in Malawi: a cost-effectiveness analysis. BMJ Open 2017;7:e015344. doi:10.1136/ bmjopen-2016-015344

- Prepublication history and additional material is available. To view please visit the journal (http://dx.doi.org/ 10.1136/ bmjopen-2016-015344).

Received 30 November 2016 Revised 20 May 2017 Accepted 22 May 2017

CrossMark

${ }^{1}$ Department of Pediatrics, University of California, San Francisco, California, USA ${ }^{2}$ Philip R Lee Institute for Health Policy Studies, University of California, San Francisco,

California, USA

${ }^{3}$ Health Strategies International, Oakland, USA

${ }^{4}$ Philip R. Lee Institute for Health Policy Studies and Global Health Economics Consortium, University of California, San Francisco, California, USA

Correspondence to

Dr Teresa Bleakly Kortz; teresa. bleakly@ucsf.edu

\section{ABSTRACT}

Objectives Pneumonia is the largest infectious cause of death in children under 5 years globally, and limited resource settings bear an overwhelming proportion of this disease burden. Bubble continuous positive airway pressure (bCPAP), an accepted supportive therapy, is often thought of as cost-prohibitive in these settings. We hypothesise that bCPAP is a cost-effective intervention in a limited resource setting and this study aims to determine the cost-effectiveness of bCPAP, using Malawi as an example.

Design Cost-effectiveness analysis.

Setting District and central hospitals in Malawi.

Participants Children aged 1 month-5years with severe pneumonia, as defined by WHO criteria.

Interventions Using a decision tree analysis, we compared standard of care (including low-flow oxygen and antibiotics) to standard of care plus bCPAP.

Primary and secondary outcome measures For each treatment arm, we determined the costs, clinical outcomes and averted disability-adjusted life years (DALYs). We assigned input values from a review of the literature, including applicable clinical trials, and calculated an incremental cost-effectiveness ratio (ICER).

Results In the base case analysis, the cost of bCPAP per patient was $\$ 15$ per day and $\$ 41$ per hospitalisation, with an incremental net cost of $\$ 64$ per pneumonia episode. bCPAP averts 5.0 DALYs per child treated, with an ICER of $\$ 12.88$ per DALY averted compared with standard of care. In one-way sensitivity analyses, the most influential uncertainties were case fatality rates (ICER range \$9-32 per DALY averted). In a multi-way sensitivity analysis, the median ICER was $\$ 12.97$ per DALY averted $(90 \% \mathrm{CI}$, $\$ 12.77$ to $\$ 12.99$ ).

Conclusion bCPAP is a cost-effective intervention for severe paediatric pneumonia in Malawi. These results may be used to inform policy decisions, including support for widespread use of bCPAP in similar settings.

\section{INTRODUCTION}

In 2015, over 5.9 million children worldwide died before their fifth birthday; the majority of these deaths were preventable or treatable with simple, inexpensive interventions. ${ }^{1}$ The leading infectious cause of death in children under 5 years is pneumonia, accounting for
Strengths and limitations of this study

- Only cost-effectiveness analysis evaluating the use of bubble continuous airway pressure (bCPAP) for paediatric pneumonia.

- We chose an example low-income country (Malawi) where costing and outcomes data exist.

- In general, we used conservative estimates that would overestimate bCPAP costs and underestimate benefits, and the intervention was still cost-effective.

- Because of extensive sensitivity analyses, we are confident that our results are robust.

- Cost-effectiveness analyses are inherently limited by the data available.

- Most individual inputs are based on a single study, generally with a small sample size.

- The case fatality rate for standard of care and bCPAP came from a randomised controlled trial in Bangladesh and were determined using the proxy of treatment failure rates as opposed to reported mortality rates given Malawi's more limited resources. The case fatality/treatment failure rates from the Bangladeshi trial are supported by results from prospective cohort studies conducted in Malawi.

- The cost of long-term sequelae is a rough estimate based on the cost of lifelong treatment, which likely overestimates the cost considerably.

$15 \%$ of paediatric deaths worldwide, and resource-limited

settings bear a disproportionate share of mortality and disease burden. ${ }^{2}$ Pneumonia frequently causes respiratory distress and hypoxia in children, which can lead to respiratory failure and cardiac arrest in severe or untreated cases. The highest case fatality rate (CFR) occurs in children with severe pneumonia (table 1 ). ${ }^{34}$ Even a small improvement in the management of pneumonia could result in a significant decrease in childhood morbidity and mortality.

Effective bubble continuous positive airway pressure (bCPAP) reduces the need 
Table 1 WHO classification of pneumonia for children aged 2-59 months by severity of disease ${ }^{15}$

\begin{tabular}{ll}
\hline Diagnosis & Presenting signs and symptoms \\
\hline Pneumonia & Fast breathing (>50bpmages \\
& $2-11$ months, $>40$ bpmages $1-5$ years) \\
& Chest indrawing \\
Severe pneumonia & Cough or difficulty in breathing with: \\
& Oxygen saturation $<90 \%$ or central \\
& cyanosis \\
& Severe respiratory distress (eg, \\
& grunting and very severe chest \\
& indrawing) \\
& Signs of pneumonia with a general \\
& danger sign (inability to breastfeed \\
& or drink, lethargy or reduced level of \\
& consciousness and convulsions) \\
\hline
\end{tabular}

for invasive methods of respiratory support (intubation and mechanical ventilation), ${ }^{56}$ and has been shown to improve clinical outcomes in several resource-limited settings: India, Malawi, Ghana, Vietnam and Bangladesh to name a few. ${ }^{6-11}$ However, bCPAP is not universally available despite compelling evidence of its benefits, possibly because it is deemed too expensive for resource-limited settings.
Malawi is a low-income, HIV-endemic country in southern Africa with limited resources and a high burden of disease: 43000 children under 5 years died in 2012 alone, ${ }^{2}$ and pneumonia continues to be the leading cause of childhood death with a $24.3 \%$ annual incidence rate ${ }^{4}$ and a CFR of $23.1 \%$ in children with very severe/severe pneumonia. $^{3} 1213$

Our review of the literature yielded few cost-effectiveness analyses of bCPAP in the treatment of pneumonia in resource-limited settings, and no analyses of bCPAP in severe pneumonia in a paediatric, non-neonatal, population. This study addresses this gap in knowledge with the following aims: (1) to quantify the clinical benefits of bCPAP in the treatment of severe paediatric pneumonia in Malawi as measured by mortality rates and disability-adjusted life years (DALYs), (2) to assess the costs associated with implementation of bCPAP in Malawi, and (3) to determine the incremental cost-effectiveness ratio (ICER) of bCPAP as compared with standard of care.

\section{METHODS}

\section{Overview}

The focus of this study is children under 5 years, excluding neonates, in Malawi with severe pneumonia, by WHO criteria. ${ }^{14}$ We constructed a decision tree with Microsoft Excel for Mac 2011, V.4.4.3 comparing current standard of
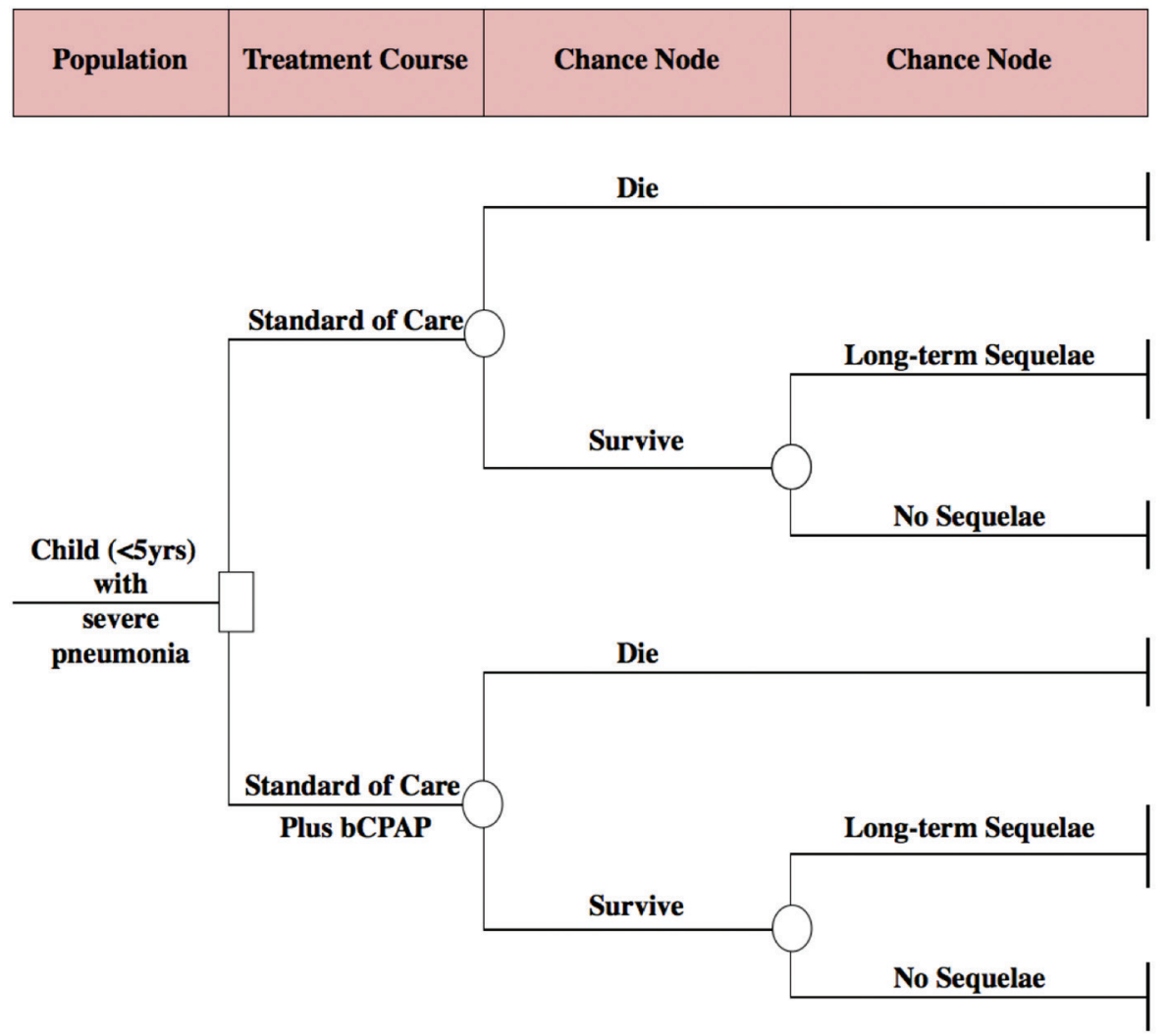

Figure 1 Decision tree depicting the two treatment options (standard of care and standard of care plus bCPAP) for severe paediatric pneumonia in Malawi. bCPAP, bubble continuous positive airway pressure. 
care with standard of care plus bCPAP (figure 1, detailed decision tree available in online supplementary figure 1A). The standard of care in Malawi for the treatment of severe paediatric pneumonia includes hospitalisation at a district or central hospital with a dedicated paediatric ward, antibiotic therapy, and oxygen therapy via an oxygen concentrator and nasal cannula in a high-dependency unit. ${ }^{12}$

\section{Intervention}

Treatment for severe paediatric pneumonia ideally includes six elements: provider knowledge to appropriately manage pneumonia; oxygen; antibiotics; non-invasive positive pressure ventilation (such as bCPAP); non-invasive monitoring (continuous pulse oximetry); and nasopharyngeal (NP) suctioning. The first three are part of standard of care in Malawi. For bCPAP delivery, we modelled our analysis on a basic, modified nasal prong and oxygen concentrator model, ${ }^{15}$ a bCPAP system previously shown to be effective in treating severe pneumonia in children in resource-limited settings. ${ }^{78}$ For bCPAP, we also included the costs of provider training, pulse oximetry and NP suction as these are integral to the intervention.

\section{Analytic approach}

We took the perspective of a Malawian government hospital, encompassing all (ie, societal) direct medical costs, with a lifelong horizon in terms of morbidity and mortality. The benefit of averted mortality is the discounted average life expectancy, while the cost of longterm sequelae is the discounted cost of lifelong therapy.

\section{Inputs and assumptions}

Cost inputs came from published values in the literature or vendors (online supplementary table 1A). We identified resources required for bCPAP from prior microcosting studies in Malawi. ${ }^{12}{ }^{16}$ Specific indirect provider training costs are allocated for the implementation of bCPAP and based on published costs associated with the Child Lung Health Programme (CLHP) in Malawi. The CLHP trained providers in the diagnosis and treatment of pneumonia and the use of oxygen therapy. ${ }^{3}{ }^{13}$ The CLHP also supplied oxygen concentrators and essential supplies to 25 paediatric wards around the country. ${ }^{12}$ We included the cost of essential capital equipment: an additional oxygen concentrator, pulse oximeter and NP suctioning device. We assumed the oxygen concentrator would be used for bCPAP for 90 days out of the year, and also assumed no additional benefit when not in use for bCPAP. The entire bCPAP system, including the concentrator, reusable components, pulse oximeter, NP suction device and spare parts, has a lifespan of 5 years.

We did not include extra personnel time in the bCPAP intervention as there are limited data on the extra time required, and based on conversations with providers from this setting, we assume it to be minimal. Prior analysts have made the same assumption. ${ }^{16}{ }^{17}$ We used activity unit costs and relied on data from WHO-CHOosing Interventions that are Cost-Effective to determine the average cost per bed day in a public teaching hospital in Malawi. ${ }^{18}$ In addition to bed-day costs, we included the cost of antibiotics, a chest radiograph and laboratory investigations in the cost of hospitalisation. The range for vendor costs used in sensitivity analysis was set at $\pm 50 \%$.

Survival and sequelae probabilities were determined through review of the literature. CFRs for both bCPAP and standard of care came from a single, randomised controlled trial (RCT) conducted in Bangladesh with three treatment arms: low-flow oxygen, high-flow oxygen and bCPAP. ${ }^{7}$ In this RCT, patients who failed low-flow oxygen were then randomised to high-flow oxygen or bCPAP therapy, and those who failed bCPAP or high-flow oxygen were intubated and mechanically ventilated. ${ }^{7}$ In Malawi, high-flow oxygen, bCPAP and mechanical ventilation are not routinely available as rescue therapies. For this reason, we chose to use treatment failure rates as a proxy for mortality. When reliable studies were unavailable, educated assumptions were made and noted as estimates. We used the WHO and Global Burden of Disease published disability weights for treated or untreated lower respiratory tract infection (LRTI) for children ${ }^{19}$ and accounted for the risk of long-term sequelae in survivors. ${ }^{20}$ Complication rates of bCPAP in prior studies have been reported as negligible or non-existent; therefore, we did not include an input for bCPAP-related complications. ${ }^{21-24}$ All costs are reported in US\$ adjusted for inflation based on the Consumer Price Index. We discounted health outcomes (death and DALYs) and costs by $3 \%$.

We calculated DALYs following a patient from birth with an average age of onset of severe pneumonia of 1 year $^{5}$ and an average life expectancy if one survives to age 5 of 65.4 years. ${ }^{25}$ Long-term sequelae of pneumonia include: restrictive lung disease, obstructive lung disease, bronchiectasis, chronic bronchitis, asthma and abnormal pulmonary function or chronic respiratory disease not otherwise specified. ${ }^{20}$ Most of these conditions are chronically controlled with a combination of an inhaled steroid and a $\beta_{2}$-agonist. The Global Asthma Network recommends beclomethasone (steroid) and salbutamol $\left(\beta_{2}\right.$-agonist $)$ in resource-limited settings, ${ }^{26}$ and both are listed in the Malawian Standard Treatment Guidelines published by the Ministry of Health. ${ }^{27}$ We assumed that sequelae are life-long and non-progressive and an affected person requires daily medications to control symptoms and prevent acute exacerbations. We used data from resource-limited settings for length of stay (LOS) for pneumonia survivors and non-survivors with bCPAP $^{19}$ and without it, ${ }^{3728}$ as well as for average duration of bCPAP therapy. ${ }^{67}$

We assigned baseline values and ranges to each health outcome and cost input based on confidence intervals or plausible ranges as determined from review of the literature (table 2). Each input is an estimate based on the best sources available. We used a series of deterministic 
Table 2 Base case input values and ranges as supported by the literature and used in the decision tree analysis

\begin{tabular}{|c|c|c|c|c|}
\hline Input & Base case value & $\begin{array}{l}\text { Published } \\
\text { range }\end{array}$ & $\begin{array}{l}\text { Sensitivity } \\
\text { parameter estimate } \\
\text { (min, max) }\end{array}$ & Source \\
\hline \multicolumn{5}{|l|}{ Health input } \\
\hline Standard of care CFR & 0.24 & $0.12-0.24$ & $(0.12,0.36)$ & $\begin{array}{l}\text { Chisti et } a l^{7} \\
\text { Enarson et al } \\
\text { Lazzerini et }\left.a\right|^{30}\end{array}$ \\
\hline bCPAP CFR & 0.06 & $0.04-0.12$ & $(0.01,0.12)$ & Chisti et al $l^{7}$ \\
\hline Risk of long-term sequelae & 0.14 & $0.06-0.21$ & $(0.06,0.21)$ & Edmond et $a l^{20}$ \\
\hline $\begin{array}{l}\text { Disability weight per episode of treated/ } \\
\text { untreated LRTI for children }\end{array}$ & 0.28 & $\mathrm{n} / \mathrm{a}$ & $(0.14,0.42)$ & WHO $2015^{19}$ \\
\hline $\begin{array}{l}\text { Disability weight for chronic sequelae of } \\
\text { treated/untreated LRTI for children }{ }^{19}\end{array}$ & 0.1 & $\mathrm{n} / \mathrm{a}$ & $(0.05,0.15)$ & WHO $2015^{19}$ \\
\hline \multicolumn{5}{|l|}{ Cost input ${ }^{\star}$} \\
\hline $\begin{array}{l}\text { Daily costs for bCPAP } \\
\text { (US } \$ / \text { per patient day) }\end{array}$ & $\$ 15.41$ & $\mathrm{n} / \mathrm{a}$ & $(7.70,23.11)$ & Composite \\
\hline $\begin{array}{l}\text { One-time costs for bCPAP } \\
\text { (US\$/per patient hospitalisation) }\end{array}$ & $\$ 10.57$ & $\mathrm{n} / \mathrm{a}$ & $(5.29,15.86)$ & Composite \\
\hline $\begin{array}{l}\text { Daily cost of inpatient hospital care } \\
\text { (US\$/per patient day) }\end{array}$ & $\$ 4.48$ & $\mathrm{n} / \mathrm{a}$ & $(2.24,6.72)$ & $\begin{array}{l}\text { WHO-CHOICE } \\
\text { MSH }^{35}\end{array}$ \\
\hline $\begin{array}{l}\text { One-time costs of inpatient hospital care } \\
\text { (US } \$ / \text { per patient hospitalisation) }\end{array}$ & $\$ 5.10$ & $0-6.64$ & $(2.55,7.65)$ & Ayieko $2009^{36}$ \\
\hline $\begin{array}{l}\text { Cost of long-term sequelae } \\
\text { (US } \$ / \text { per episode) }\end{array}$ & $\$ 656.43$ & $\mathrm{n} / \mathrm{a}$ & $(328.22,984.65)$ & WHO-CHOICE MSH ${ }^{18}$ \\
\hline $\begin{array}{l}\text { LOS if patient dies: low-flow oxygen } \\
\text { (days) }\end{array}$ & 1 & $1-2$ & $(0,2)$ & Chisti et al ${ }^{7}$ \\
\hline LOS if patient dies: bCPAP (days) & 2 & $1-3$ & $(1,3)$ & Chisti et $a l^{7}$ \\
\hline $\begin{array}{l}\text { LOS if patient survives: low-flow oxygen } \\
\text { (days) }\end{array}$ & 4 & $3-6$ & $(2,6)$ & $\begin{array}{l}\text { Chisti et } \mathrm{al}^{7} \\
\text { Chola and Robberstad }{ }^{28} \\
\text { Enarson et } \mathrm{al}^{3}\end{array}$ \\
\hline LOS if patient survives: bCPAP (days) & 5 & $3-7$ & $(3,8)$ & $\begin{array}{l}\text { Chisti et al } \\
\text { Jayashree } 2016^{21}\end{array}$ \\
\hline bCPAP duration (days) & 2 & $1-3$ & $(1,3)$ & $\begin{array}{l}\text { Chisti et al }{ }^{7} \\
\text { Kinikar et } a l^{6}\end{array}$ \\
\hline
\end{tabular}

Sensitivity analysis parameters are $0.5(\mathrm{~min})$ and $1.5(\max )$ times the base case value.

${ }^{*}$ Net present value is the total adjusted cost based on the Consumer Price Index (2015 US\$) with discounting (3\%) when appropriate.

CFR, case fatality rate; LOS, length of stay; n/a, not applicable; WHO-CHOICE, CHOosing Interventions that are Cost-Effective.

one-way (Microsoft Excel) and multi-way probabilistic (@ Risk Palisade software, V.6.3.1: Industrial version) sensitivity analyses, assuming uniform distributions and extreme but plausible values for the parameters of all inputs, to evaluate the effect of uncertainty on each of the model's important cost and health inputs on the ICER.

\section{RESULTS}

bCPAP costs $\$ 15$ per patient day and $\$ 41$ per hospitalisation. The increased probability of survival resulted in added hospital days. The base case analysis shows that the cost of treating one child with severe pneumonia is $\$ 88$ for standard of care and $\$ 152$ for standard of care plus bCPAP. This yields an overall incremental net cost of $\$ 64$ per use of bCPAP compared with standard of care and an ICER of $\$ 12.88$ per DALY averted (table 3 ).
Standard of care and bCPAP incur an average of 7.4 and 2.4 DALYs per child treated, respectively, a difference of 5.0 DALYs.

Table 3 Cost-effectiveness results by treatment course

\begin{tabular}{|c|c|c|c|c|c|}
\hline $\begin{array}{l}\text { Treatment } \\
\text { course }\end{array}$ & $\begin{array}{l}\text { Cost } \\
\text { (US\$) }\end{array}$ & $\begin{array}{l}\text { Delta } \\
\text { cost } \\
\text { (US\$) }\end{array}$ & $\begin{array}{l}\text { DALYs } \\
\text { incurred }\end{array}$ & $\begin{array}{l}\text { DALYs } \\
\text { averted }\end{array}$ & $\begin{array}{l}\text { ICER } \\
\text { (US\$ per } \\
\text { DALY } \\
\text { averted) }\end{array}$ \\
\hline $\begin{array}{l}\text { Standard of } \\
\text { care }\end{array}$ & 88 & - & 7.4 & - & - \\
\hline bCPAP & 152 & 64 & 2.4 & 5.0 & 12.88 \\
\hline
\end{tabular}

Costs and DALYs are per patient treated.

bCPAP, bubble continuous positive airway pressure; DALYs, disability-adjusted life years; ICER, incremental cost-effectiveness ratio. 


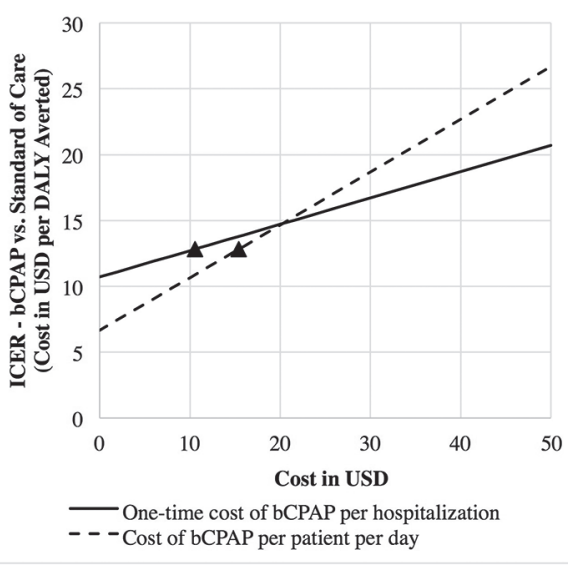

Figure 2 Variation in ICER values across a range of bCPAP treatment costs. Base case values demarcated with a triangle. bCPAP, bubble continuous positive airway pressure; DALYs, disability-adjusted life years; ICER, incremental costeffectiveness ratio.

A series of one-way sensitivity analyses were performed to test key inputs across the range of input values. Variation in costs associated with bCPAP and their effect on the ICER are shown in figure 2, while variations in the CFRs for standard of care and bCPAP are shown in figure 3 .

We ranked inputs in order of effect on the median ICER; the inputs causing the greatest variability were CFRs for standard of care and bCPAP, cost per day for bCPAP, and bCPAP duration. All inputs, including those pertaining to the intervention-CFR for bCPAP, duration of bCPAP, cost of bCPAP per day and one-time costs for bCPAP-influenced the median ICER between $\$ 9$ and $\$ 40$ per DALY averted (figure 4 ). The multi-way probabilistic analysis resulted in a median ICER of $\$ 12.97$ per

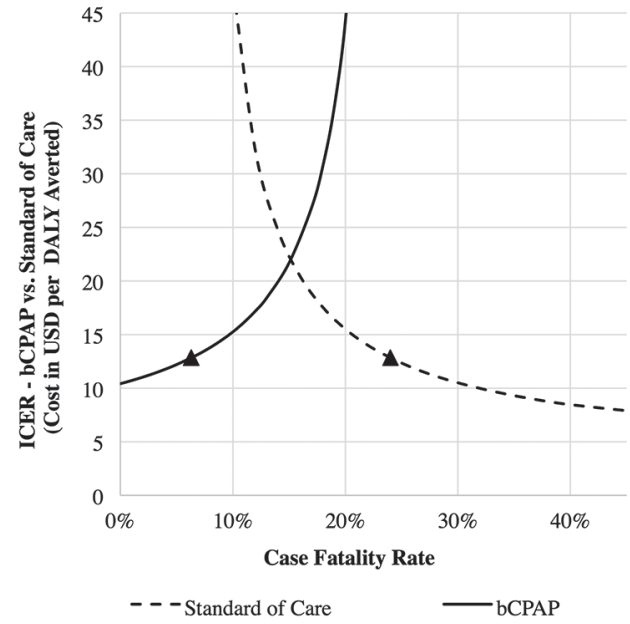

Figure 3 Variation in ICER as CFR varies in the two treatment arms: standard of care and standard of care plus bCPAP. The CFR in one arm is held constant while the other is varied. Base case values demarcated with a triangle. bCPAP, bubble continuous positive airway pressure; CFR, case fatality rate; DALYs, disability-adjusted life years; ICER, incremental cost-effectiveness ratio.
DALY averted (90\% CI, $\$ 12.77$ to $\$ 12.99$; online supplementary figure $2 \mathrm{~A}$ ).

\section{DISCUSSION}

Our base case analysis demonstrated an ICER of $\$ 12.88$ per DALY averted, which is highly cost-effective by most standards. National immunisation programmes in resource-limited settings cost approximately $\$ 7-\$ 438$ per DALY averted. ${ }^{29}$ Multi-way sensitivity analyses produced a median ICER close to the base case and a narrow CI. The inputs that caused the greatest median ICER variability were CFRs for standard of care and bCPAP, daily bCPAP costs and LOS. LOS directly impacted the cost of hospitalisation and indirectly affected the cost of bCPAP; bCPAP lengthened LOS through increased survival for children who would otherwise have died, which was accounted for in this model. bCPAP therapy would need to extend LOS considerably longer than standard of care to create an unfavourable ICER, and there is no evidence for this in the literature.

CFRs were highly influential in this model. We used treatment failure rates from Chisti $e t a l$ as a surrogate for mortality. ${ }^{7}$ The CFR for standard of care was consistent with data from Malawi reported by Enarson et al, though higher than reported in an observational study by Lazzerini et al (CFR for severe pneumonia by WHO criteria was $21.9 \%-23.1 \%$ and $11.8 \%$, respectively). ${ }^{3}{ }^{30}$ If we used the published CFRs by Chisti et al (3.8\% for bCPAP and 14.9\% for low-flow) or the standard of care CFR from Lazzerini et al then the base case ICER would be $\$ 22.50$ or $\$ 33.30$ per DALY averted, respectively, still cost-effective by most standards.

Our findings are consistent with past studies of similar interventions. In Papua New Guinea, oxygen therapy was cost-effective with an ICER of $\$ 50$ per DALY averted, ${ }^{31}$ and in Malawi, bCPAP was cost-effective for neonates with an ICER of $\$ 4.20$ per life year gained. ${ }^{16}$ The latter study by Chen et al appears more favourable than our results, but there are several notable differences in cost inputs: we accounted for training costs, maintenance costs, the cost of pulse oximetry and the cost for NP suction. When these additional costs are taken into account, our results are consistent with that of Chen $e t a l^{16}$

There are several limitations to this analysis. Most individual inputs are based on a single study, generally with a small sample size. The CFR for standard of care and bCPAP came from an RCT in Bangladesh ${ }^{7}$; we chose to use failure rates as a proxy for mortality due to treatment arm crossover and a lack of rescue therapies, namely mechanical ventilation, in Malawi. It is possible that the failure rates overestimate the CFR in both arms; however, the standard of care CFR is supported by results from prospective cohort studies conducted in Malawi, ${ }^{3}{ }^{30}$ though similar corroborating results do not exist for the bCPAP CFR in Malawi. Our sensitivity analyses examined wide ranges for both mortality rates and included rates beyond what is currently published. The cost of long-term 


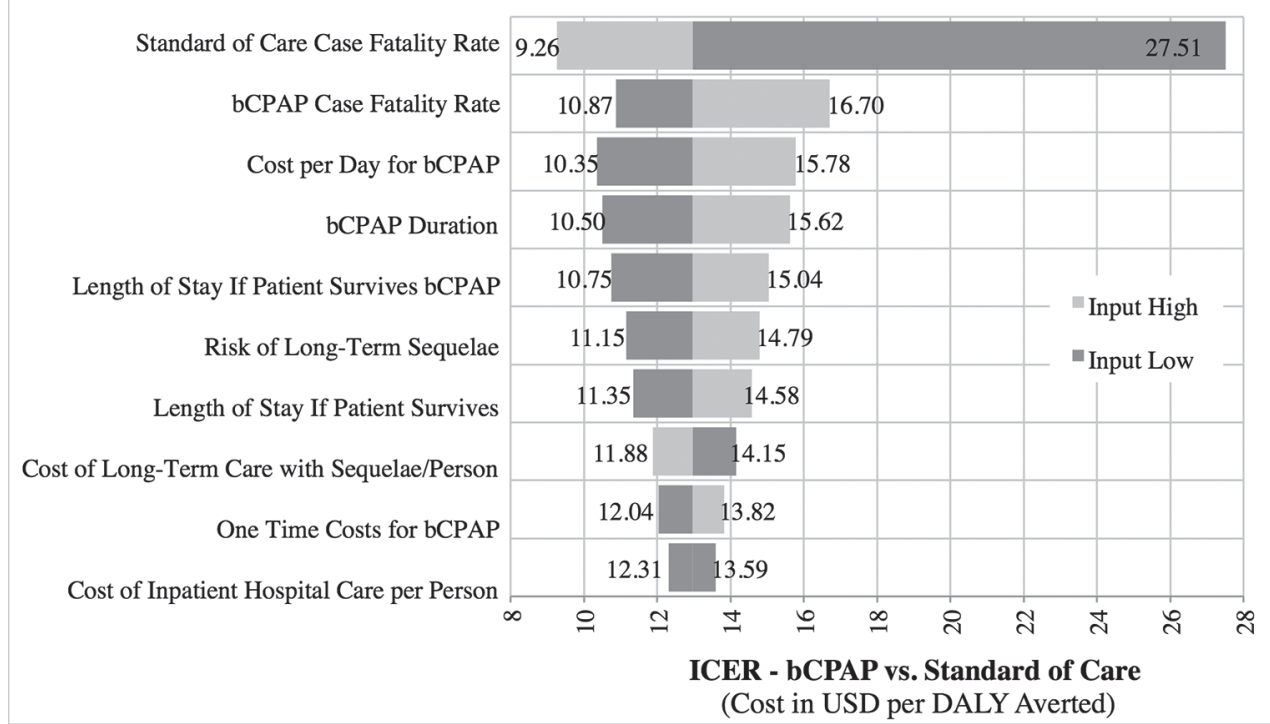

Figure 4 Tornado plot for the multi-way probabilistic sensitivity analysis demonstrating inputs with the greatest impact on median ICER value variability. bCPAP, bubble continuous positive airway pressure; DALYs, disability-adjusted life years; ICER, incremental cost-effectiveness ratio.

sequelae is a rough estimate based on the cost of lifelong treatment with a recommended inhaled steroid and a $\beta_{2}$-agonist; however, our estimate likely overestimates the cost as not all patients with sequelae will need or be prescribed therapy, and overall access to affordable medications in Malawi is poor. ${ }^{32}$ Extensive sensitivity analyses were performed in an attempt to account for the imprecision in the model, and our finding of excellent cost-effectiveness is robust.

In general, we used conservative estimates that would overestimate bCPAP costs and underestimate benefits. This includes the assumption that bCPAP would be used for 90 days out of the year and only for the treatment of pneumonia. bCPAP is also an effective supportive therapy for sepsis, anaemia, dengue and shock, ${ }^{11}$ which are not accounted for in this model. Added use of bCPAP would disperse fixed costs more widely. We modelled the cost of training, but no additional benefit, though skilled providers identify and manage patients more effectively. ${ }^{33}$ Much of the overall cost of bCPAP can be attributed to additional hospital costs and, in part, to long-term sequelae due to increased survival. Overall, we believe that bCPAP may be more cost-effective than our model shows.

It is far more meaningful to estimate costs and effectiveness within the local context of disease burden and available resources ${ }^{34}$ as opposed to assigning an arbitrary cost-effectiveness threshold. This analysis indicates that bCPAP for severe paediatric pneumonia can be life saving and cost-effective in resource-limited settings similar to that of Malawi. An estimated $95 \%$ of all episodes of clinical pneumonia are in resource-limited settings: if every child under 5 years with severe pneumonia had access to effective bCPAP, the worldwide pneumonia mortality rate would decrease by $33 \% .^{27}$
When considering whether to introduce a new bCPAP device as compared with using an oxygen concentrator, ${ }^{16}$ we were concerned about a possible unintended consequence; one oxygen concentrator with tubing can be 'split' to provide low-flow oxygen for up to four children at once. If the concentrator is used instead for bCPAP, which requires higher flow rates, only one patient can receive treatment per concentrator, leaving potentially three other patients without oxygen. We do not recommend that oxygen concentrators be used for bCPAP at the expense of children needing low-flow oxygen as this would deny children standard of care. This is why we included the cost of an oxygen concentrator in our model, though we recognise that this does not completely eliminate this allocation dilemma in settings with an insufficient number of concentrators.

The cost-effectiveness analysis is an analytical tool that adds data-in this instance favourable data-regarding the value of the implementation of interventions in relevant settings (for bCPAP, resource-limited contexts similar to Malawi). Much of the current global health funding is devoted to the introduction of new technologies, as opposed to focusing on wide implementation of already available, effective and inexpensive therapies. We found that the existing bCPAP technology is not only appropriate, but also cost-effective and life saving for the treatment of severe pneumonia in resource-limited settings. Malawi is primed for a nationwide roll out of bCPAP with modest investment from a donor or the Ministry of Health given the existing equipment, training and infrastructure. bCPAP applicability in other countries will need to be assessed, and implementation tailored to available resources and priorities. The results of this study support widespread implementation of bCPAP in Malawi, and potentially in similar resource-limited settings, which 
could greatly decrease childhood morbidity and mortality globally.

Acknowledgements The authors thank Sarah Myers, Hans-Joerg Lang and Rebecca Richards-Kortum for their expertise, guidance and correspondence.

Contributors TBK, JGK and EM: designed the study. TBK: collected the data, performed the literature search and constructed the decision tree. TBK and $\mathrm{BH}$ : performed the sensitivity analyses, wrote the manuscript and generated the figures and tables, which were edited by JGK and EM. All authors: interpreted the data and were involved in the decision to submit the manuscript for publication.

Competing interests None declared.

Provenance and peer review Not commissioned; externally peer reviewed.

Data sharing statement We, as the authors of this original research article, state that there are no additional, unpublished data available from this study.

Open Access This is an Open Access article distributed in accordance with the Creative Commons Attribution Non Commercial (CC BY-NC 4.0) license, which permits others to distribute, remix, adapt, build upon this work non-commercially, and license their derivative works on different terms, provided the original work is properly cited and the use is non-commercial. See: http://creativecommons.org/ licenses/by-nc/4.0/

(c) Article author(s) (or their employer(s) unless otherwise stated in the text of the article) 2017. All rights reserved. No commercial use is permitted unless otherwise expressly granted.

\section{REFERENCES}

1. United Nations International Children's Emergency Fund. Committing to Child Survival: A Promise Renewed. Progress Report 2015. New York, NY: UNICEF, 2015.

2. World Health Organization. World Health Statistics: 2014. Geneva, Switzerland, 2014.

3. Enarson PM, Gie RP, Mwansambo CC, et al. Reducing deaths from severe pneumonia in children in Malawi by improving delivery of pneumonia case management. PLoS One 2014;9:e102955.

4. Rudan I, O'Brien KL, Nair H, et al. Epidemiology and etiology of childhood pneumonia in 2010: estimates of incidence, severe morbidity, mortality, underlying risk factors and causative pathogens for 192 countries. J Glob Health 2013;3:010401.

5. Bahman-Bijari B, Malekiyan A, Niknafs P, et al. Bubble-CPAP vs Ventilatory-CPAP in preterm infants with respiratory distress. Iran $J$ Pediatr 2011;21:151-8.

6. Kinikar A, Kulkarni R, Valvi C, et al. Use of indigenous bubble CPAP during swine flu pandemic in Pune, India. Indian J Pediatr 2011;78:1216-20.

7. Chisti MJ, Salam MA, Smith JH, et al. Bubble continuous positive airway pressure for children with severe pneumonia and hypoxaemia in Bangladesh: an open, randomised controlled trial. Lancet 2015;386:1057-65.

8. Jayashree M, KiranBabu HB, Singhi S, et al. Use of Nasal Bubble CPAP in Children with Hypoxemic Clinical Pneumonia-Report from a Resource Limited Set-Up. J Trop Pediatr 2016;62:69-74.

9. Kawaza K, Machen HE, Brown J, et al. Efficacy of a low-cost bubble CPAP system in treatment of respiratory distress in a neonatal ward in Malawi. PLoS One 2014;9:e86327.

10. Wilson PT, Morris MC, Biagas KV, et al. A randomized clinical trial evaluating nasal continuous positive airway pressure for acute respiratory distress in a developing country. J Pediatr 2013;162:988-92.

11. Cam BV, Tuan DT, Fonsmark L, et al. Randomized comparison of oxygen mask treatment vs nasal continuous positive airway pressure in dengue shock syndrome with acute respiratory failure. $J$ Trop Pediatr 2002;48:335-9.

12. Enarson P, La Vincente S, Gie R, et al. Implementation of an oxygen concentrator system in district hospital paediatric wards throughout Malawi. Bull World Health Organ 2008;86:344-8.

13. Enarson PM, Gie R, Enarson DA, et al. Development and implementation of a national programme for the management of severe and very severe pneumonia in children in Malawi. PLoS Med 2009;6:e1000137.
14. World Health Organization. Revised WHO classification and treatment of pneumonia in children at health facilities: evidence summaries. Geneva, Switzerland: World Health Organization, 2014.

15. Duke T. CPAP: a guide for clinicians in developing countries. Paediatr Int Child Health 2014;34:3-11.

16. Chen A, Deshmukh AA, Richards-Kortum R, et al. Cost-effectiveness analysis of a low-cost bubble CPAP device in providing ventilatory support for neonates in Malawi - a preliminary report. BMC Pediatr 2014;14:288

17. Duke T, Peel D, Wandi F, et al. Oxygen supplies for hospitals in papua new guinea: a comparison of the feasibility and costeffectiveness of methods for different settings. P N G Med $J$ 2010;53:126-38.

18. World Health Organization. Cost effectiveness and strategic planning (WHO-CHOICE). 2014. http://www.who.int/choice/en/. (accessed 30 June 2016)

19. World Health Organization. Health statistics and information systems: national burden of disease supplementary files - Disability weights. 2015. http://www.who.int/healthinfo/global_burden_disease/tools_ national/en/. (accessed 15 June 2015).

20. Edmond K, Scott S, Korczak V, et al. Long term sequelae from childhood pneumonia; systematic review and meta-analysis. PLOS One 2012; 7:e31239.

21. Jayashree M, KiranBabu HB, Singhi S, et al. Use of nasal bubble CPAP in children with hypoxemic clinical pneumonia-report from a resource limited set-up. J Trop Pediatr 2016;62:69-74.

22. Wilson PT, Morris MC, Biagas KV, et al. A randomized clinical trial evaluating nasal continuous positive airway pressure for acute respiratory distress in a developing country. $J$ Pediatr 2013;162:988-92.

23. Cam BV, Tuan DT, Fonsmark L, et al. Randomized comparison of oxygen mask treatment vs. nasal continuous positive airway pressure in dengue shock syndrome with acute respiratory failure. J Trop Pediatr 2002;48:335-9.

24. Thia LP, McKenzie SA, Blyth TP, et al. Randomised controlled trial of nasal continuous positive airways pressure (CPAP) in bronchiolitis. Arch Dis Child 2008;93:45-7.

25. World Health Organization. Global Health Observatory Data Repository: life table by country - Malawi. 2015. http://apps.who.int/ gho/data/view.main.60980?lang=en. (accessed 20July 2015)

26. Robison JA, Ahmad ZP, Nosek CA, et al. Decreased pediatric hospital mortality after an intervention to improve emergency care in Lilongwe, Malawi. Pediatrics 2012;130:e676-e682.

27. Malawi Ministry of Health. Malawi Standard treatment guidelines. 4th ed. Malawi: Lilongwe, 2008.

28. Chola L, Robberstad B. Estimating average inpatient and outpatient costs and childhood pneumonia and diarrhoea treatment costs in an urban health centre in Zambia. Cost Eff Resour Alloc 2009;7:16.

29. Brenzel L, Wolfson LJ, Fox-Rushby J, et al. Vaccine-Preventable Disease. In: Jamison DT, Breman JG, Measham AR, eds. Disease Control Priorities in developing countries. 2nd ed. New York: Oxford University Press, 2006:389-412.

30. Lazzerini M, Seward N, Lufesi N, et al. Mortality and its risk factors in Malawian children admitted to hospital with clinical pneumonia, 2001-12: a retrospective observational study. Lancet Glob Health 2016:4:e57-e68.

31. Duke T, Wandi F, Jonathan M, et al. Improved oxygen systems for childhood pneumonia: a multihospital effectiveness study in Papua New Guinea. Lancet 2008;372:1328-33.

32. Mendis S, Fukino K, Cameron A, et al. The availability and affordability of selected essential medicines for chronic diseases in six low- and middle-income countries. Bull World Health Organ 2007;85:279-88.

33. Irimu GW, Gathara D, Zurovac D, et al. Performance of health workers in the management of seriously sick children at a Kenyan tertiary hospital: before and after a training intervention. PLoS One 2012;7:e39964

34. Marseille E, Larson B, Kazi DS, et al. Thresholds for the costeffectiveness of interventions: alternative approaches. Bull World Health Organ 2015;93:118-24.

35. Management Sciences for Health (MSH). International Drug Price Indicator Guide, 2014 Edition. MSH Medford,Mass, 2015.

36. Ayieko P, Akumu AO, Griffiths UK, et al. The economic burden of inpatient paediatric care in Kenya: household and provider costs for treatment of pneumonia, malaria and meningitis. Cost Eff Resour Alloc 2009;7:3. 